\title{
Histone modifications change with age, dietary restriction and rapamycin treatment in mouse brain
}

\author{
Huan Gong ${ }^{1,5}$, Hong Qian ${ }^{1}$, Robin Ertl ${ }^{2,6}$, Clinton M. Astle ${ }^{2}$, Gang G. Wang ${ }^{3}$, David E. \\ Harrison ${ }^{2}$ and Xiangru $X \mathbf{u}^{1,4}$ \\ ${ }^{1}$ Department of Anesthesiology, Yale University School of Medicine, New Haven, CT, USA \\ 2 The Jackson Laboratory, Bar Harbor, ME, USA \\ ${ }^{3}$ Department of Biochemistry and Biophysics and Lineberger Comprehensive Cancer Center, University of North Carolina at \\ Chapel Hill, Chapel Hill, NC, USA \\ ${ }^{4}$ Max Planck Institute for Biology of Ageing, Cologne, Germany \\ ${ }^{5}$ The Key Laboratory of Geriatrics, Beijing Institute of Geriatrics, Beijing Hospital, Ministry of Health, Beijing, China \\ ${ }^{6}$ Center for Natural and Health Sciences, Marywood University, Scranton, PA, USA \\ Correspondence to: Xiangru $\mathrm{X} U$, email: xiangru.xu@yale.edu \\ David E. Harrison, email: david.harrison@jax.org
}

Keywords: gerotarget, gerontology, health span, mTOR, rapalogs

Received: April 17, $2015 \quad$ Accepted: April 21, 2015

Published: May 20, 2015

This is an open-access article distributed under the terms of the Creative Commons Attribution License, which permits unrestricted use, distribution, and reproduction in any medium, provided the original author and source are credited.

\section{ABSTRACT}

The risk of developing neurodegenerative disorders such as Alzheimer's disease (AD) increases dramatically with age. Understanding the underlying mechanisms of brain aging is crucial for developing preventative and/or therapeutic approaches for age-associated neurological diseases. Recently, it has been suggested that epigenetic factors, such as histone modifications, maybe be involved in brain aging and agerelated neurodegenerations. In this study, we investigated 14 histone modifications in brains of a cohort of young ( 3 months), old ( 22 months), and old age-matched dietary restricted (DR) and rapamycin treated BALB/c mice. Results showed that 7 out of all measured histone markers were changed drastically with age. Intriguingly, histone methylations in brain tissues, including H3K27me3, H3R2me2, H3K79me3 and $\mathrm{H} 4 \mathrm{~K} 20 \mathrm{me} 2$ tend to disappear with age but can be partially restored by both DR and rapamycin treatment. However, both DR and rapamycin treatment also have a significant impact on several other histone modifications such as H3K27ac, H4K16ac, H4R3me2, and H3K56ac, which do not change as animal ages. This study provides the first evidence that a broad spectrum of histone modifications may be involved in brain aging. Besides, this study suggests that both DR and rapamycin may slow aging process in mouse brain via these underlying epigenetic mechanisms.

\section{INTRODUCTION}

In industrialized countries, life expectancy is rapidly rising, and this has led to an increasing incidence of agerelated neurodegenerative disorders including Alzheimer's disease (AD), Parkinson disease (PD) and Huntington Disease (HD). The lack of effective therapies that can halt and/or reverse the progression of these disorders creates an enormous burden on both affected individuals and society as a whole. Developing preventative or therapeutic interventions for such conditions demands deeper understanding of the processes underlying normal brain aging [1-2]. Brain aging is marked by a gradual decline in cognitive function, which is often correlated with age-dependent deterioration of synaptic function in brain regions crucial for memory formation and consolidation, such as the hippocampus and prefrontal cortex [3-4]. The neurobiological processes underlying these age-related learning and memory deficits include aberrant changes in gene transcription that eventually affects the plasticity of the aged brain. Changes in gene expression in neurons were thought to take place during 
brain aging, and analysis of regions of the hippocampus and front cortex by microarray has confirmed this [5-7]. The molecular mechanisms underlying these changes in gene expression and the regulation are largely unknown, but recent studies point to a novel possibility that the dysregulation of epigenetic control of gene expression may be involved [8-10].

The term "epigenetics" was first coined by Conrad Waddington in the 1940s to describe interactions of genes with their environment during development stages [11]. The first suggestion that DNA methylation might play an important biological role was made by Griffith and Mahler in 1969 indicating that it could provide a basis for long term memory in the brain [12]. The two most widely studied epigenetic codes are DNA methylation and histone modifications. Epigenetic regulation is a key transcriptional mechanism that alters gene expression by altering the structure and/or conformation of chromatin but does not change the basic genetic code. Many fundamental cellular processes are affected by epigenetic modulation, and it has become evident that chromatin-based epigenetic mechanisms underlie important aspects of the aging process. Recent reports indicate that chromatin remodeling via histone acetylation plays a crucial role in regulating synaptic and cognitive function in aging and neurodegenerative brains [13-16]. Increasing histone acetylation by inhibition of histone deacetylase (HDAC) enhances gene transcription and improves hippocampal long-term potentiation (LTP) or memory functions in several experimental models of neurological diseases [1719], indicating that dysregulation of chromatin acetylation may be involved in certain forms of cognitive impairment [20-21]. Histone methylation can occur on either lysine $(\mathrm{K})$ or arginine $(\mathrm{R})$ residues, and can be mono-, di- or trimethylated, and either activation or repression is dependent upon the particular histone residue, that is modified [22-23]. Therefore, identifying new histone modifications that may be involved in brain aging has a potential to provide critical insights into the neurobiology of aging and possibly the etiology of neurodegenerative disorders.

Dietary restriction (DR) delays aging and improves resistance to disease in a fashion that is evolutionarily conserved from yeast to primates and humans [24-25], though controversy was stirred lately on an NIH-initiated primate cohort study [26]. These beneficial effects include, in mammals, the attenuation of age-associated cognitive impairment and neurodegeneration [27-28]. More specifically, synaptic plasticity was shown to be enhanced by DR, as evidenced by increased LTP [29]. rapamycin, an antibiotic, is already in use for suppressing the immune system in transplant patients and has been used in the treatment of certain cancers for decades. Lately rapamycin treatment produced remarkable effect of extending lifespan even though it was not started until the mice had lived 600 days - equivalent to human being aged 60 years [30]. A similar benefit on lifespan has also been observed when started on the drug at a younger age (9 months old) [31]. Blagosklonny discussed the link between aging and diseases by rapamycin treatment [32]. Moreover, Rapamycin has been found to have the potential for reversing some of the effects of premature aging [33]. Interestingly, rapamycin treatment suppresses brain aging in senescence-accelerated OXYS rats [34] and results in an improvement in cognitive functions that normally decline with age in mice [35-36]. Furthermore, inhibition of mTOR by rapamycin can slow or block AD progression in a transgenic mouse model of the disease [37].

Finally, epigenetic factors are not only inheritable but also reversible. Thus, these factors, such as histone acetylation and methylation, may participate in the modulation of cell plasticity in responses to environmental cues in the brain. It is of great interest to hypothesize that intervention approaches such as DR and rapamycin administration, which not only extending the lifespan of animals under certain conditions but also promote certain brain functions in mice, may impact some ageinduced post-translational modifications of histones. In the present study, we aim to test this hypothesis by examining expression of a panel of 14 histone modifications in brains of a mouse cohort in which the mice were maintained for over 2 years either eating ad libitum, or with either DR or rapamycin treatment.

\section{RESULTS}

To identify the histone modifications which either change with age and/or respond to age-interventions including DR and rapamycin treatment in mouse brain, a cohort of BALB/c mice with groups of young (3 months), old (22 months), old age-matched DR, and old agematched rapamycin treatment and a panel of 14 histone antibodies against specific methylations or acetylations in residues of both lysine $(\mathrm{K})$ and arginine $(\mathrm{R})$ were applied in this study (Table 1). Surprisingly, over $50 \%$ of detected histone modifications in brains from these mice were altered as a result of the age of animals or the ageinterventions. Interestingly, those modifications have not been reported to be associated with brain aging or ageinterventions until now.

Among the measured histone modifications, the levels of H3K27me3, H3R2me 2 and H3K79me3 in brains of old ( 22 months) mice were reduced to $68 \%(p<0.01)$, $83 \%(p<0.05)$ and $67 \%(p<0.05)$ respectively when compared to 3 -month old animals. However, both DR and rapamycin treatment prevented the age-induced losses of H3K27me3, H3R2me2 and H3K79me3. Specifically, DR restored the levels of these histones of old age group to $92.5 \%, 132 \%$ and $104 \%$ of young age group, respectively, and likewise, rapamycin exhibited an even stronger impact by increasing the levels of these histone methylations to $133 \%, 132 \%$ and $120 \%$ of those seen in the young 
age group (Figure 1A-1C). Interestingly, the level of H4K20me2 is increased with age $(159 \%, p<0.05)$ when in mice on DR, this was reduced to $108 \%$. Rapamycin treatment, contrary to DR, was unable to suppress the ageinduced H4K20me2 expression (Figure 1D).

In addition, levels of $\mathrm{H} 3 \mathrm{~K} 18 \mathrm{ac}, \mathrm{H} 3 \mathrm{~K} 4 \mathrm{me} 2$ and $\mathrm{H} 3 \mathrm{~K} 4 \mathrm{me}$ were also changed with brain aging (Figure 2 A-C). The levels of $\mathrm{H} 3 \mathrm{~K} 18 \mathrm{ac}$ and $\mathrm{H} 3 \mathrm{~K} 4 \mathrm{me} 2$ increased $44 \%(\mathrm{p}<0.05)$ and $29 \%(p<0.01)$, respectively, and the level of $\mathrm{H} 3 \mathrm{~K} 4 \mathrm{me}$ decreased $32 \%(\mathrm{p}<0.01)$ in old brains when compared to that of young brains. The levels of $\mathrm{H} 3 \mathrm{~K} 18 \mathrm{ac}$ and $\mathrm{H} 3 \mathrm{~K} 4 \mathrm{me} 2$ were enhanced further by DR
(76\%, $p<0.05$ and 44\%, $p<0.01$, respectively) and rapamycin $(77 \%, p<0.01$ and $16 \%, p<0.05$, respectively) when compared to the old group. Furthermore, the level of $\mathrm{H} 3 \mathrm{~K} 4 \mathrm{me}$ declined with age and was further diminished by both DR and rapamycin $(51 \%$ and $70 \%, p<0.01$, respectively), when compared with the old group.

Both DR and rapamycin also demonstrated unique functions in modulating the expression of histone modifications that do not respond to age. Four transcriptional activation histone modifications, H3K27ac, H4K16ac, H4R3me2 and H3K56ac, do not shown detectable differences between young and old brains, but
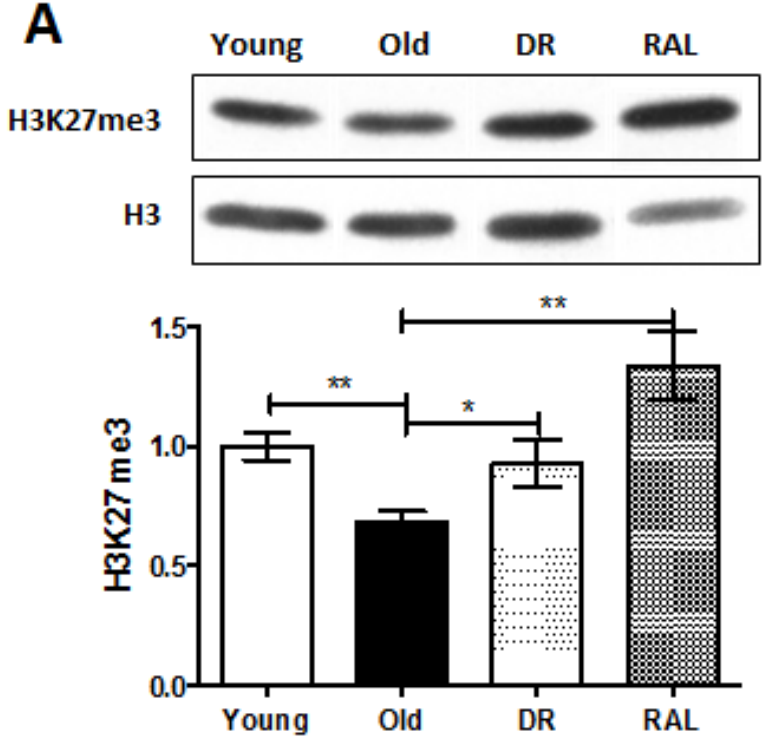

C
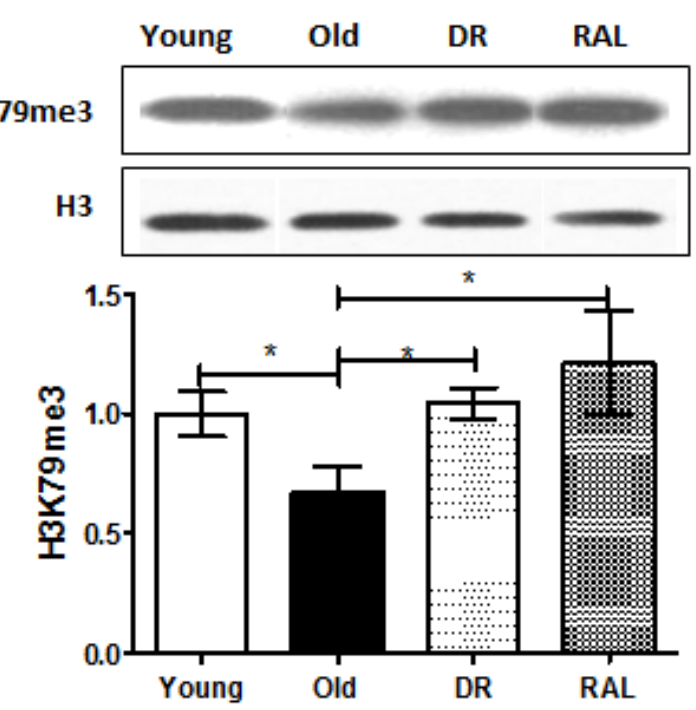

B

H3R2me2

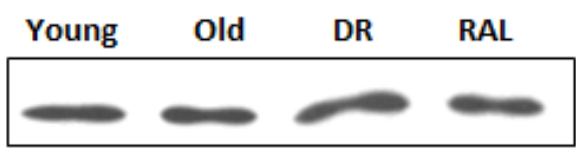

H3
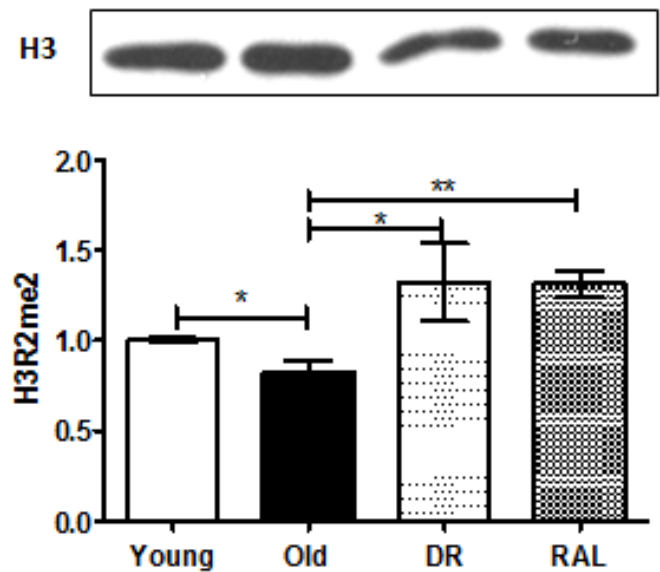

D
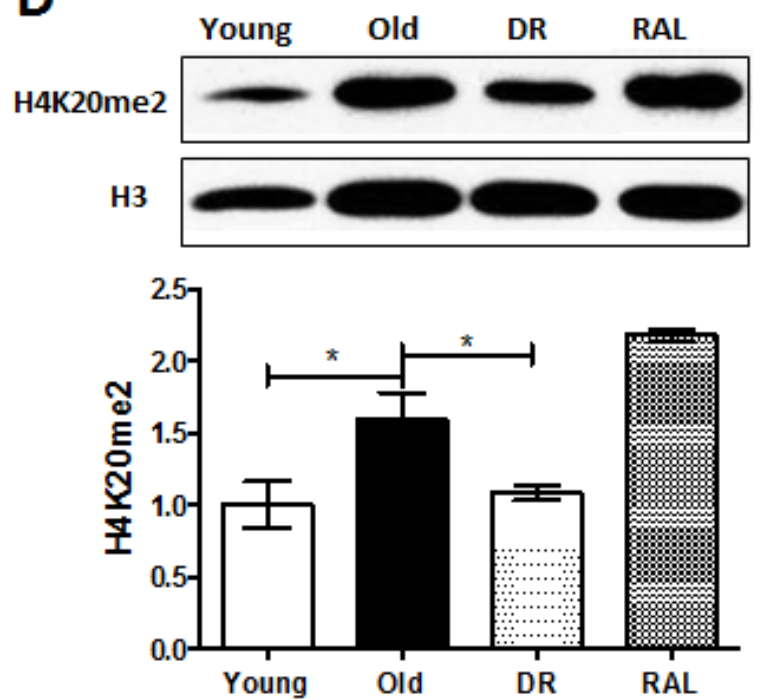

Figure 1: Histone methylations are changed with age but the age-associated change is prevented by $D R$ and/or rapamycin treatment in mouse brain. Representative western blots (upper) and quantitative densitometric analysis (lower) of H3K27me3 A., H3R2me2 B., H3K79me3 C. and H4K20me2 D. in brains from 3 months old ad libitum (Young), 22 months old ad libitum (Old) , 22 months old dietary restricted (DR) and 22 months old rapamycin treatment (RAL) BALB/c mice. Histone modification levels were normalized to total $\mathrm{H} 3$ content. The values (mean \pm S.E.M.) are expressed as a percentage relative to the 3 months old group. $n=3 \sim 5$ animals per goup . $(* * p<0.01, * p<0.05$ Old versus Young, DR versus Old, RAL versus Old). 
their expression was changed significantly by DR and/or rapamycin treatment (Figure 2D-2G). Compared to the age-matched controls, both DR and rapamycin treatment increased the expression of $37 \%$ and $54 \%$, respectively of H3K27ac $(p<0.05)$; DR alone decreased expression of H4K16ac to $69 \%(p<0.05)$. Rapamycin increased expression of H4R3me 2 to $188 \%$ but decreased the expression of H3K 56 ac over 10 times $(p<0.05)$.

\section{DISCUSSION}

The organization of the eukaryotic genome into chromatin enables DNA to fit inside the nucleus. Essentially, histone proteins form a "spool" which allows DNA to be wound around them. Post-translational modification of histones has an effect on the tightness of DNA winding; it thus regulates the the accessibility of proteins such as transcriptional activators/repressors to
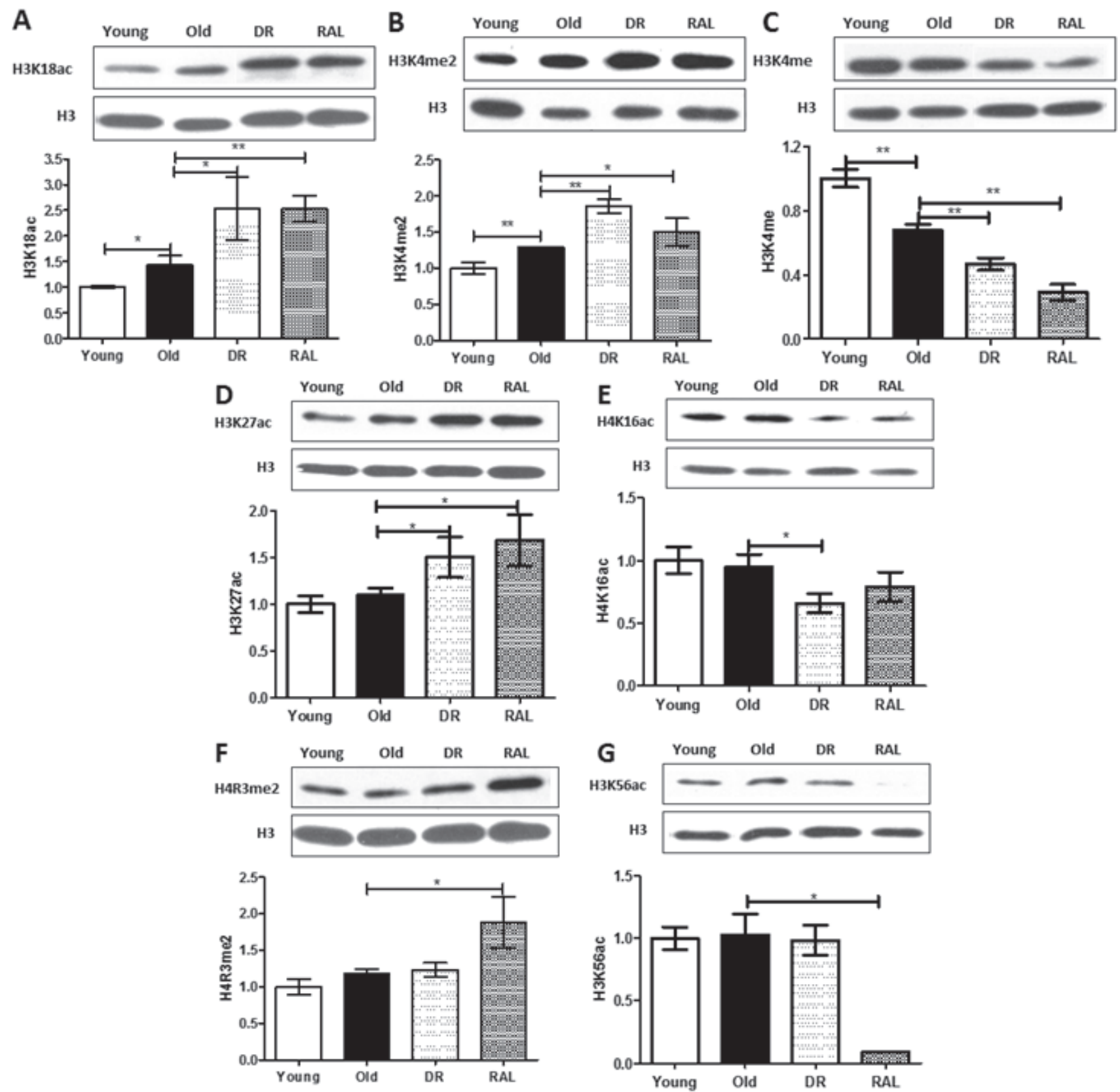

Figure 2: Distinctive impact of DR and/or rapamycin treatment on histone modifications in mouse brain. Representative western blots (upper) and quantitative densitometric analysis (lower) of H3K18ac A., H3K4me2 B., H3K4me C., H3K27ac D., H4K16ac E., H4R3me2 F. and H3K56ac G. in brains from 3 months old ad libitum (Young), 22 months old ad libitum (Old), 22 months old dietary restricted (DR) and 22 months old rapamycin treatment (RAL) BALB/c mice. Histone modification levels were normalized to total H3 content. The values (mean \pm S.E.M.) are expressed as a percentage relative to the 3 months old group. $n=3 \sim 5$ animals per goup . (** $p<$ $0.01, * p<0.05$ Old versus Young, DR versus Old, RAL versus Old). 
Table 1. Summarization of measured histones in BALB/c brains

\begin{tabular}{c|c|c|c|}
\hline Antibodieslexperiment & young vs old & DR vs old & RAL vs old \\
H3R2me2 & $\searrow$ & $\nearrow$ & $\nearrow$ \\
H3K27me3 & $\searrow$ & $\nearrow$ & $\nearrow$ \\
H3K79me3 & $\searrow$ & $\nearrow$ & $\nearrow$ \\
\hline H4K20me2 & $\nearrow$ & $\searrow$ & $\rightarrow$ \\
\hline H3K4me & $\searrow$ & $\searrow$ & $\searrow$ \\
H3K4me2 & $\nearrow$ & $\nearrow$ & $\nearrow$ \\
H3K18ac & $\nearrow$ & $\nearrow$ & $\nearrow$ \\
H4K16ac & $\rightarrow$ & $\searrow$ & $\rightarrow$ \\
H3K27ac & $\rightarrow$ & $\nearrow$ & $\nearrow$ \\
H3K56ac & $\rightarrow$ & $\rightarrow$ & $\searrow$ \\
H4R3me2 & $\rightarrow$ & $\rightarrow$ & $\nearrow$ \\
H4K20me & $\rightarrow$ & $\rightarrow$ & $\rightarrow$ \\
H3K4me3 & $\rightarrow$ & $\rightarrow$ & $\rightarrow$ \\
H3K9me3 & $\rightarrow$ & $\rightarrow$ & $\rightarrow$ \\
* $\$ reduced expression & $\nearrow$ increased expression $\rightarrow$ no change
\end{tabular}

the DNA to facilitate genomic functions by turning on and off of gene expression. Tight winding keeps genes switched off, while loosening the packaging allows gene to be turned on. Therefore, remodeling of chromatin by histone modifications, such as acetylation, methylation and phosphorylation, is a key mechanism in controlling gene transcription in a variety of important biological processes [38].
Recent work suggests that histone acetylation has a critical role in age-associated declines in cognitive functions. Rodents exhibit a transient increase in histone acetylation after exposure to different learning paradigms [9]. Restoration of the expression of H4K12ac in mouse brain leads to a recovery of cognitive functions [16]. More importantly, rodents, after treatment with histone deacetylase inhibitors (HDACis), have demonstrated that

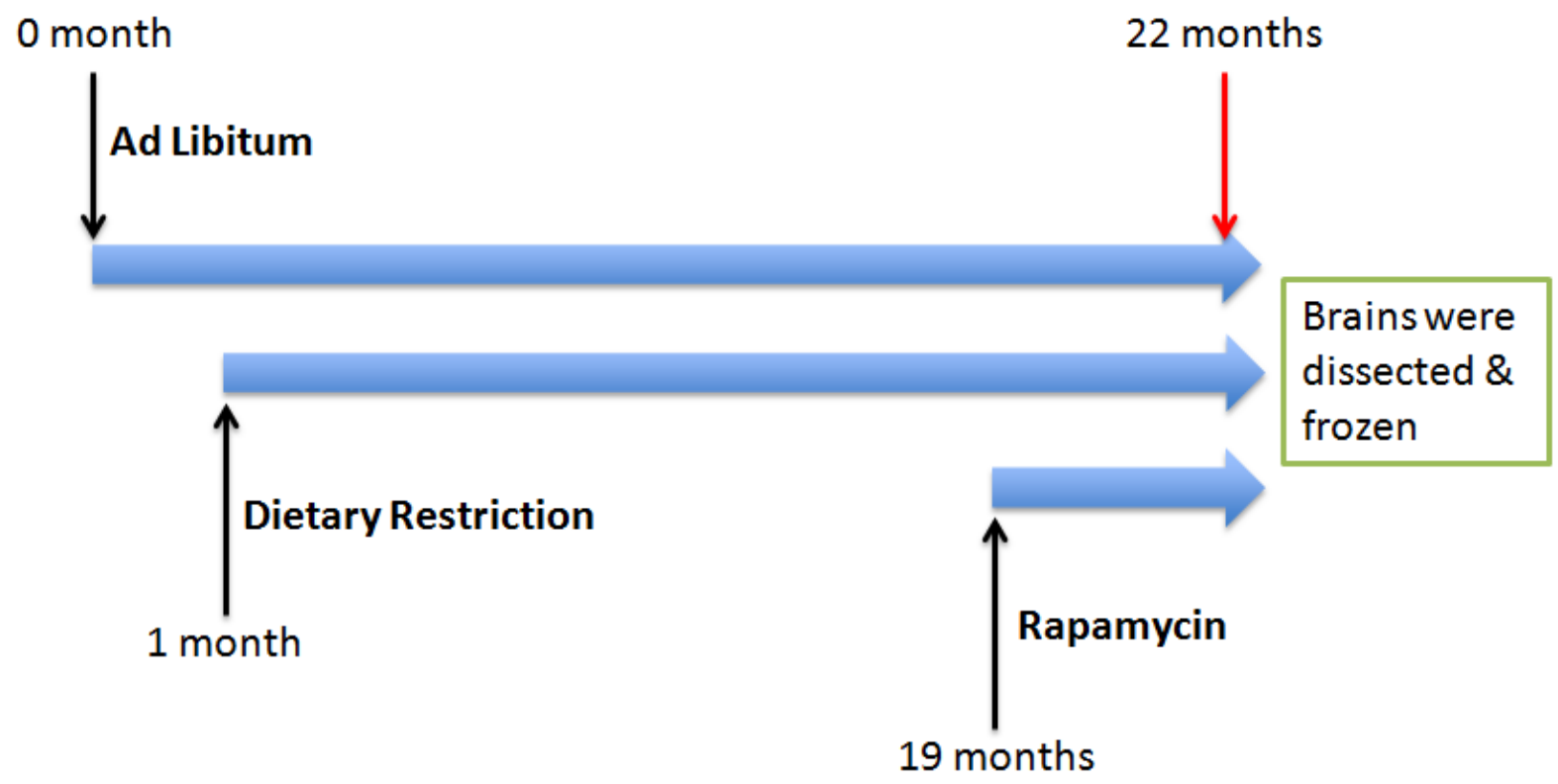

Figure 3: Experimental design. A cohort of BALB/C mice were subjected to ad libitum, Dietary restriction and rapamycin at various time points. 
targeting histone acetylation has emerged as a promising strategy for therapeutic intervention in age-related cognitive function decline and neurodegenerative diseases $[17,39]$.

Histone methylation is another important and widespread type of chromatin modification that is known to influence biological processes in the context of development and cellular responses [21]. Histone methylation can occur on either lysine $(\mathrm{K})$ or arginine $(\mathrm{R})$ residues, and these can be mono-, di- or trimethylated, and either activation or repression is dependent upon the particular lysine residue that is modified [21]. For instance, $\mathrm{H} 3 \mathrm{~K} 4 \mathrm{me} 3$, an active mark for transcription, is upregulated in hippocampus one hour following contextual fear conditioning [40]. Interestingly, a line of exciting evidence indicates that specific histone methylations, H3K4me3 and $\mathrm{H} 3 \mathrm{~K} 27 \mathrm{me} 3$, may act as a regulator of lifespan was confirmed by studies in C. elegans [41-42]. In addition, studies have shown that the expression of p16INK4a, a cell-cycle regulatory protein and an aging biomarker, can be regulated by $\mathrm{H} 3 \mathrm{~K} 27 \mathrm{me} 3$ during cellular senescence [43]. However, whether histone methylation is involved in brain aging is still an open question. Encouragingly, we identified alterations in the methylation level of several hisotone residues including $\mathrm{H} 3 \mathrm{~K} 27 \mathrm{me} 3, \mathrm{H} 3 \mathrm{R} 2 \mathrm{me} 2$, H3K79me3 and H4K20me2 with aging of mouse brains (Figure 1). The arginine residue methylation of Histone 3, H3R2me2, has never been associated with any brain function previously. More interestingly, these age-associated alterations are prevented by DR and/or rapamycin treatment (Figure 1). However, further work needs to be done to (1) study the functional significance of these histone changes in mouse brain aging, and (2) identify the transcriptional targets of these age-associated histone markers in neuronal cells.

Brain aging and its direct consequences, such as degenerative diseases and even death, are inevitable; however, scientific advances in understanding basic aging mechanisms have made it much more feasible to postpone aging processes and to increase the animal lifespan using some specific regimens including DR and rapamycin administration. DR has been studied for decades in extending lifespans across species but the underlying molecular mechanism is not fully clear yet [24, 27]. Rapamycin, a bacterial product first isolated from soil on Easter Island, is the first drug intervention to reliably increase mammalian lifespan by $10 \%$ or more [30-31]. More intriguingly, Kolosova et. al. first described that rapamycin prevents brain aging in rat [34]; rapamycin also produces an improvement in age-associated cognitive functions in mouse[35-37]. We therefore wondered if epigenetic modifications play a role in mediating the beneficial effects of both DR and rapamycin in mouse brain aging. The results from our study unexpectedly exhibited that both DR and rapamycin can restore, at least partially, the age-related alterations in histone methylation levels (Figure 1). This may put forward a novel epigenetic mechanism of beneficial age-interventions. However, it is still not known whether these epigenetic responses have any relevance with the classic DR-driven IGF/Insulin and rapamycin-driven mTOR pathways in the brain. In addition, age and DR or rapamycin exhibit similar effects on the overall level of several histone modifications, such as H3K18ac, H3K4me2 and H3K4me (Figure 2A2C). It implies that those histone modifications may play dual regulatory roles in mediating both age and age-interventions. One possible favorable explanation is that age-adaptive cellular stress responses could be enhanced by DR and/or rapamycin to strengthen neuronal networks and plasticity [44]. Our study also showed that histone modifications such as $\mathrm{H} 4 \mathrm{~K} 16 \mathrm{ac}$ and $\mathrm{H} 3 \mathrm{~K} 56 \mathrm{ac}$ were stable with age but regulated by DR or rapamycin treatment (Figure 2D-2G). Interestingly, H4K16ac has been identified as DR regulated histone marker that may be modulated by Sirt1 regulation [45]. H3K56ac has also been identified biochemically as a direct target of mTOR inhibition by a chemical genomic screening recently [46]. The latter suggests a novel chromatin-regulating role for mTOR signaling.

In summary, this study has examined a panel of histone modifications in mouse brain, and different patterns of histone modifications are responsive to normal aging and age-interventions including DR and rapamycin treatment. Based on these findings, we propose that dysregulation of chromatin remodeling may occur and contribute to brain aging as well as cognitive impairments observed in age-related neurodegenerative diseases such as AD. Promising interventions targeting age-related conditions may prevent the associated change of histone modifications or bypass age-induced cognitive deficit with compensational pathways. We know that a large amount of careful work needs to be carried out in the future uncover the functional significance of these promising histone modifications. We hope this study offers enriched epigenetic information to brain aging and neurodegeneration researchers and encourages the field to consider and understand the role of epigenetic regulation in neuroplasticity and cognitive function.

\section{MATERIALS AND METHODS}

\section{Animals}

BALB/C female mice were produced and maintained at the Jackson Laboratory (Bar Harbor, ME). At weaning ( 3 weeks of age), they were housed 4 per box in weaning cages. These cages were assigned to one of 3 feeding regimens using Purina LabDiet's 5LG6 irradiated formulation of the NIH-31 (4\% fat) diet. Groups of ad libitum (AL) fed mice, both young (3 months) and old 
(22 months), were given uninhibited access to grain. A Group of DR mice were fed with $70 \%$ of the AL feeding rate after weaning and continued lifelong (22 months of age). For group of rapamycin-treated mice, the rapamycin was encapsulated, and $2.24 \mathrm{mg}$ of rapamycin per $\mathrm{kg}$ body weight/day was administered (equivalent to $\mathrm{AL}$ feeding) for 3 months starting when the animals were 19 months of age [30]. All procedures were carried out as indicated in Figure 3 and approved by the animal care and use committee of the Jackson Laboratory, and staff veterinarians monitored mice on a regular basis, finding no pathogens.

\section{Tissues preparation}

Mice were sacrificed by cervical dislocation according to the approved protocol. Then the health status of mice was first checked anatomically. The whole brains including all major parts of the brain of all mice without liver/kidney problems, severe inflammations' or tumors that could impact brain health (e.g., pituitary tumors) were dissected out and snap frozen in liquid nitrogen, ground into powder with mortar and pestle in liquid nitrogen and then stored at $-80^{\circ} \mathrm{C}$.

\section{Acid extraction of histones}

Core histone proteins were extracted from the mouse whole brain samples using an acid extraction method as described by Shechter et al. with slight modifications [47]. Briefly, all procedures were performed on ice, and all solutions were chilled to $4^{\circ} \mathrm{C}$ before use unless otherwise indicated. All centrifugation steps were performed at $4^{\circ} \mathrm{C}$. Thirty to sixty microgram whole brain powder was homogenized in ice-cold PBS containing the following: $1 \mathrm{mM}$ phenylmethylsulfonyl fluoride, $1 \mathrm{x}$ complete protease inhibitor cocktail (Roche, cat. no. 1697498), 5mM sodium butyrate (Sigma-Aldrich, B5887). The homogenates were centrifuged at $1000 \mathrm{~g}$ for $10 \mathrm{~min}$ and the supernatant was discarded. The pellet was then resuspended in $1 \mathrm{ml}$ hypotonic lysis buffer (10mM Tris- $\mathrm{Cl}$ pH 8.0, $1 \mathrm{mM} \mathrm{KCl}$, $1.5 \mathrm{mM} \mathrm{MgCl} 2,1 \mathrm{mM}$ DTT), incubated for $30 \mathrm{~min}$ on rotator at $4^{\circ} \mathrm{C}$, centrifuged at $14,000 \mathrm{~g}$ for $10 \mathrm{~min}$, and the supernatant (cytoplasmic fraction) was aspirated. The pellet (nuclear fraction) was then resuspended in $0.5 \mathrm{ml}$ of $0.4 \mathrm{~N} \mathrm{H} 2 \mathrm{SO} 4$, incubated for $30 \mathrm{~min}$ to overnight and centrifuged at $16,000 \mathrm{~g}$ for $10 \mathrm{~min}$. The supernatant was transferred to a fresh tube, and proteins were precipitated with acetone. Acid extracted histone proteins were then collected by centrifugation at $16,000 \mathrm{~g}$ for $10 \mathrm{~min}$. The supernatant was discarded, and the protein pellet was washed with acetone. The resulting purified histone proteins were resuspended in deionized water and stored at $-80^{\circ} \mathrm{C}$ until processed for Western blotting.

\section{Western blotting and quantification}

The proteins in sample buffer were denatured by keeping at $100^{\circ} \mathrm{C}$ for 3-5 min in a dry bath. About $1 \sim 5 \mathrm{ug}$ of histone proteins were separated in a $14 \%(\mathrm{w} / \mathrm{v})$ SDSPAGE and transferred onto a nitrocellulose membrane (Bio-rad). After blocking the membrane with 5\% (W/V) BSA in Tris-Buffered Saline Tween-20 (TBST), the membrane was incubated overnight with primary antibody, washed in TBST, incubated with Horseradish peroxidase (HRP) conjugated secondary antibody (antirabbit IgG, Sigma; anti-mouse IgG, Upstate) at 1:5,000 dilution in TBST, washed and carefully developed with ECL (Pierce). The following primary antibodies were used: H3(ab 1791, Abcam), H3K4me3(39159, Active Motif), H3R2me2(04-808, UBI), H3K27me3 (39156, Active Motif), H3K79me3 (ab 2621, Abcam), H4K20me (ab 9051, Abcam), H3K56ac(07-677, UBI), H3K9me3(39161, Active Motif), H4K20me2(07-367, UBI), H4K16ac(39167, Active Motif), H3K18ac (ab 1191, Abcam), H4R3me2(ab 5823, Abcam), H3K27ac (39685, Active Motif), H3K4me(ab 8895,Abcam), H3K4me2(07-030, UBI). The quality, especially the specificity of all antibodies was first verified experimentally by us (data not shown). The blot was scanned by using Gel Doc XR system (Bio-Rad) with a dynamic range of greater than 3 order of magnitude, and signal intensities were quantified using Image J (http:// rsb.info.nih.gov/ij/), a most popular image acquisition tool for accurately calculating area and pixel value, and normalized to values of total $\mathrm{H} 3$, which is widely used as a loading control to quantify the amount of modifications of histones.

\section{Statistical analysis}

Statistical analyses were performed using SPSS statistics software. One-way ANOVA was used to evaluate the $p$-value among comparisons, and then followed with a further post-hoc multiple comparisons between groups according to the equal variances assumed or not. Results were presented as group means \pm SEM. Each group has 3-5 animals. Statistical significance was defined as $p<0.05$.

\section{ACKNOWLEDGMENTS}

We are grateful to Dr. Niklason for the thoughtful discussion and advice. This work was supported by Yale University, NIH (X.X. AT008679-01) and National Natural Science Foundation of China (G. H. No. 81300693).

\section{CONFLICTS OF INTEREST}

The authors declare no conflict of interest. 


\section{Editorial note}

This paper has been accepted based in part on peerreview conducted by another journal and the authors' response and revisions as well as expedited peer-review in Oncotarget.

\section{REFERENCES}

1. Bishop NA, Lu T and Yankner BA. Neural mechanisms of ageing and cognitive decline. Nature, 2010. 464: p. 529-35.

2. Costa G and Di Milia L. Aging and shift work: a complex problem to face. Chronobiol Int, 2008. 25: p. 165-81.

3. Tapia-Arancibia L, Aliaga E, Silhol M, Arancibia S. New insights into brain BDNF function in normal aging and Alzheimer disease. Brain Res Rev, 2008. 59: p. 201-20.

4. Lynch G, Rex CS and Gall CM. Synaptic plasticity in early aging. Ageing Res Rev, 2006. 5: p. 255-80.

5. Xu X., Zhan M, Duan W, Prabhu V, Brenneman R, Wood W, Firman J, Li H, Zhang P, Ibe C, Zonderman AB, Longo DL, Poosala S, et al. Gene expression atlas of the mouse central nervous system: impact and interactions of age, energy intake and gender. Genome Biol, 2007. 8: p. R234.

6. Xu X. Single Cell Transcriptome Study in Brain Aging. Single Cell Biol, 2012. 1: p. el11.

7. Rowe WB, Blalock EM, Chen KC, Kadish I, Wang D, Barrett JE, Thibault O, Porter NM, Rose GM, Landfield PW.et al., Hippocampal expression analyses reveal selective association of immediate-early, neuroenergetic, and myelinogenic pathways with cognitive impairment in aged rats. J Neurosci, 2007. 27: p. 3098-110.

8. Roth TL, Roth ED and Sweatt JD. Epigenetic regulation of genes in learning and memory. Essays Biochem, 2010. 48: p. 263-74.

9. Kosik KS, Rapp PR, Raz N, Small SA, Sweatt JD, Tsai LH. Mechanisms of age-related cognitive change and targets for intervention: epigenetics. J Gerontol A Biol Sci Med Sci, 2012. 67: p. 741-6.

10. Lubin FD, Gupta S, Parrish RR, Grissom NM, Davis RL. Epigenetic mechanisms: critical contributors to long-term memory formation. Neuroscientist, 2011. 17: p. 616-32.

11. Van Speybroeck L. From epigenesis to epigenetics: the case of C. H. Waddington. Ann N Y Acad Sci, 2002. 981: p. 6181.

12. Griffith JS and Mahler HR. DNA ticketing theory of memory. Nature, 1969. 223: p. 580-2.

13. Haggarty SJ and Tsai LH. Probing the role of HDACs and mechanisms of chromatin-mediated neuroplasticity. Neurobiol Learn Mem, 2011. 96: p. 41-52.

14. Zeng Y, Tan M, Kohyama J, Sneddon M, Watson JB, Sun YE, Xie CW. Epigenetic enhancement of BDNF signaling rescues synaptic plasticity in aging. J Neurosci, 2011. 31: p. 17800-10.
15. Levenson JM and Sweatt JD. Epigenetic mechanisms in memory formation. Nat Rev Neurosci, 2005. 6: p. 108-18.

16. Peleg S, Sananbenesi F, Zovoilis A, Burkhardt S, BahariJavan S, Agis-Balboa RC, Cota P, Wittnam JL, GogolDoering A, Opitz L, Salinas-Riester G, Dettenhofer M, Kang H, et al. Altered histone acetylation is associated with age-dependent memory impairment in mice. Science, 2010. 328: p. 753-756.

17. Fischer A, Sananbenesi F, Mungenast A, Tsai LH. Targeting the correct HDAC (s) to treat cognitive disorders. Trends in pharmacological sciences, 2010. 31: p. 605-617.

18. Vecsey CG, Hawk JD, Lattal KM, Stein JM, Fabian SA, Attner MA, Cabrera SM, McDonough CB, Brindle PK, Abel T, Wood MA. Histone deacetylase inhibitors enhance memory and synaptic plasticity via CREB:CBP-dependent transcriptional activation. J Neurosci, 2007. 27: p. 6128-40.

19. Francis YI, Fà M, Ashraf $H$, Zhang $H$, Staniszewski A, Latchman DS, Arancio O. Dysregulation of histone acetylation in the APP/PS1 mouse model of Alzheimer's disease. J Alzheimers Dis, 2009. 18: p. 131-9.

20. Strahl BD and Allis CD. The language of covalent histone modifications. Nature, 2000. 403(6765): p. 41-5.

21. Greer EL and Shi Y. Histone methylation: a dynamic mark in health, disease and inheritance. Nat Rev Genet, 2012. 13: p. 343-57.

22. Kouzarides T. Histone methylation in transcriptional control. Curr Opin Genet Dev, 2002. 12: p. 198-209.

23. Fischle $\mathrm{W}$, Wang $\mathrm{Y}$ and Allis CD. Histone and chromatin cross-talk. Curr Opin Cell Biol, 2003. 15: p. 172-83.

24. Fontana L, Partridge L, and Longo VD. Extending healthy life span-from yeast to humans. science, 2010. 328: p. 321-326.

25. Flurkey K, Astle CM, and Harrison DE. Life extension by diet restriction and $\mathrm{N}$-acetyl-L-cysteine in genetically heterogeneous mice. The Journals of Gerontology Series A: Biological Sciences and Medical Sciences, 2010. 65: p. 1275-1284.

26. Mattison JA, Roth GS, Beasley TM, Tilmont EM, Handy AM, Herbert RL, Longo DL, Allison DB, Young JE, Bryant M, Barnard D, Ward WF, Qi W, et al. Impact of caloric restriction on health and survival in rhesus monkeys from the NIA study. Nature, 2012. 489: p. 318-21.

27. Mattson MP. Energy intake, meal frequency, and health: a neurobiological perspective. Annu Rev Nutr, 2005. 25: p. 237-60.

28. Maalouf M, Rho JM and Mattson MP. The neuroprotective properties of calorie restriction, the ketogenic diet, and ketone bodies. Brain Res Rev, 2009. 59: p. 293-315.

29. Hori N, Hirotsu I, Davis PJ, Carpenter DO. Long-term potentiation is lost in aged rats but preserved by calorie restriction. Neuroreport, 1992. 3: p. 1085-1088.

30. Harrison DE, Strong R, Sharp ZD, Nelson JF, Astle CM, Flurkey K, Nadon NL, Wilkinson JE, Frenkel K, Carter CS, Pahor M, Javors MA, Fernandez E, et al. Rapamycin fed 
late in life extends lifespan in genetically heterogeneous mice. Nature, 2009. 460: p. 392-5.

31. Miller RA, Harrison DE, Astle CM, Baur JA, Boyd AR, de Cabo R, Fernandez E, Flurkey K, Javors MA, Nelson JF, Orihuela CJ, Pletcher S, Sharp ZD, et al., Rapamycin, but not resveratrol or simvastatin, extends life span of genetically heterogeneous mice. The Journals of Gerontology Series A: Biological Sciences and Medical Sciences, 2011. 66: p. 191-201.

32. Blagosklonny MV. Rapamycin extends life- and health span because it slows aging. Aging (Albany NY). 2013 Aug;5:592-8.

33. Ramos FJ, Chen SC, Garelick MG, Dai DF, Liao CY, Schreiber KH, MacKay VL, An EH, Strong R, Ladiges WC, Rabinovitch PS, Kaeberlein M, Kennedy BK. Rapamycin reverses elevated mTORC1 signaling in lamin A/C-deficient mice, rescues cardiac and skeletal muscle function, and extends survival. Sci Transl Med, 2012. 4: p. 144ra103.

34. Kolosova NG, Vitovtov AO, Muraleva NA, Akulov AE, Stefanova NA, Blagosklonny MV. Rapamycin suppresses brain aging in senescence-accelerated OXYS rats. Aging (Albany NY). 2013 Jun;5:474-84.

35. Halloran J, Hussong SA, Burbank R, Podlutskaya N, Fischer KE, Sloane LB, Austad SN, Strong R, Richardson A, Hart MJ, Galvan V. Chronic inhibition of mammalian target of rapamycin by rapamycin modulates cognitive and non-cognitive components of behavior throughout lifespan in mice. Neuroscience, 2012. 223: p. 102-13.

36. Majumder S, Caccamo A, Medina DX, Benavides AD, Javors MA, Kraig E, Strong R, Richardson A, Oddo $\mathrm{S}$. Lifelong rapamycin administration ameliorates agedependent cognitive deficits by reducing IL-1beta and enhancing NMDA signaling. Aging Cell, 2012. 11: p. 32635.

37. Spilman P, Podlutskaya N, Hart MJ, Debnath J, Gorostiza O, Bredesen D, Richardson A, Strong R, Galvan V. Inhibition of mTOR by rapamycin abolishes cognitive deficits and reduces amyloid-beta levels in a mouse model of Alzheimer's disease. PLoS One, 2010. 5: p. e9979.

38. Jakovcevski $\mathrm{M}$ and Akbarian S. Epigenetic mechanisms in neurological disease. Nat Med, 2012. 18(8): p. 1194-204.

39. Gräff $J$ and Tsai LH. The potential of HDAC inhibitors as cognitive enhancers. Annual review of pharmacology and toxicology, 2013. 53: p. 311-330.

40. Gupta S, Kim SY, Artis S, Molfese DL, Schumacher A, Sweatt JD, Paylor RE, Lubin FD. Histone methylation regulates memory formation. J Neurosci, 2010. 30: p. 358999.

41. Greer EL, Maures TJ, Hauswirth AG, Green EM, Leeman DS, Maro GS, Han S, Banko MR, Gozani O, Brunet A. Members of the $\mathrm{H} 3 \mathrm{~K} 4$ trimethylation complex regulate lifespan in a germline-dependent manner in C. elegans. Nature, 2010. 466: p. 383-7.
42. Maures TJ, Greer EL, Hauswirth AG, Brunet A. The H3K27 demethylase UTX-1 regulates C. elegans lifespan in a germline-independent, insulin-dependent manner. Aging Cell, 2011. 10: p. 980-90.

43. Agger K, Cloos PA, Rudkjaer L, Williams K, Andersen G, Christensen J, Helin K. The H3K27me3 demethylase JMJD3 contributes to the activation of the INK4AARF locus in response to oncogene- and stress-induced senescence. Genes Dev, 2009. 23: p. 1171-6.

44. Stranahan AM and Mattson MP. Recruiting adaptive cellular stress responses for successful brain ageing. Nat Rev Neurosci, 2012. 13: p. 209-16.

45. Li Y, Daniel M and Tollefsbol TO. Epigenetic regulation of caloric restriction in aging. BMC Med, 2011. 9: p. 98.

46. Chen H, Fan M, Pfeffer LM, Laribee RN. The histone H3 lysine 56 acetylation pathway is regulated by target of rapamycin (TOR) signaling and functions directly in ribosomal RNA biogenesis. Nucleic acids research, 2012. 40: p. 6534-6546.

47. Shechter D, Dormann HL, Allis CD, Hake SB. Extraction, purification and analysis of histones. Nat Protoc, 2007. 2: p. 1445-57. 